\begin{tabular}{|c|l|}
\hline Title & Observation and mechanism of photon emission at metal-solution interfaces \\
\hline Author(s) & Murakoshi, Kei; U osaki, Kohei \\
\hline Citation & $\begin{array}{l}\text { PHY SICAL REVIEW B, 47(4), 2278-2288 } \\
\text { https://doi.org/40.1103/PhysRevB.47.2278 }\end{array}$ \\
\hline Issue Date & 1993 \\
\hline Doc URL & http://hdl.handle.net/2115/5914 \\
\hline Rights & Copyright $\odot$ 1993 A merican Physical Society \\
\hline Type & article \\
\hline File Information & PRB47-4.pdf \\
\hline
\end{tabular}

Instructions for use 


\title{
Observation and mechanism of photon emission at metal-solution interfaces
}

\author{
Kei Murakoshi and Kohei Uosaki* \\ Physical Chemistry Laboratory, Department of Chemistry, Faculty of Science, Hokkaido University, Sapporo 060, Japan
}

(Received 4 May 1992)

\begin{abstract}
Photon emission from a metal surface $(\mathrm{Au}$ and $\mathrm{Pt}$ ) induced by electron-transfer reactions was observed in an electrolyte solution containing solvated electrons. The quantum efficiency $\Phi$ increases as the electrode potential $U_{w}$ becomes positive. The high-energy threshold of the spectrum, $E_{\mathrm{th}}$, increases linearly with increasing $U_{w}$ at the rate of $1 \mathrm{eV} / \mathrm{V}$. The relaxation energy of solvated electrons in the electrolyte solution is estimated from the values of $E_{\text {th }}$ and $U_{w}$. The peak energy $E_{p}$ of the spectrum also increases as $U_{w}$ becomes positive although the magnitude of the shift is much smaller than that of $E_{\mathrm{th}} . E_{p}$ and the low-energy threshold $E_{l}$ of the spectrum are metal dependent. The emitted-photon spectra are simulated by considering the transition between bulk band states of the metal while ignoring $\mathbf{k}$-vector conservation. The simulated results are compared with experiment and the validity of this model is discussed.
\end{abstract}

\section{INTRODUCTION}

The electron-transfer (ET) process is one of the most important elemental steps of many chemical reactions at solid-solution interfaces. Compared with the ET process in homogeneous systems, this process at solid-solution interfaces is more complicated due to the inhomogeneity of solid surfaces, the involvement of intermediate adsorbed states, and the complex electronic structure of the solid. During the past ten years, the understanding of this process has advanced quite significantly. The problem of surface inhomogeneity is almost removed by using single-crystalline metal electrodes, and the remaining inhomogeneity can be monitored by use of a scanning tunneling microscope. ${ }^{1}$ Information of the surface adsorbate can be obtained by the application of infrared reflectionabsorption spectroscopy. ${ }^{2}$ Unfortunately, however, techniques to gain knowledge of the electronic structure of a solid surface in solution is not well developed, although ultraviolet photoelectron spectroscopy (UPS) (Ref. 3) and inverse-photoemission spectroscopy (IPS) (Ref. 4) can be used to determine the occupied and unoccupied electronic states, respectively, under ultrahigh-vacuum (UHV) conditions.

In 1985, McIntyre and Sass discussed a technique called charge-transfer-reaction inverse-photoemission spectroscopy (CTRIPS) as a possible in situ technique to determine the electronic structure of electrode-electrolyte-solution interfaces. ${ }^{5-8}$ In this method, electrons are injected from high-energy chemical species in a solution to a metal via an electrochemical process and relax radiatively to unoccupied states of lower energy in the metal. Thus, the characteristics of the emitted photon are expected to reflect the electronic structure of unoccupied states of the metal in a solution. Photon emission is also induced by hole injection from an electron acceptor in solution. In the latter case, information can be obtained on the occupied states of the electrode. The CTRIPS is expected to be used to determine both the occupied and the unoccupied electronic states of the electrode in an electrolyte solution. Furthermore, since the injected electrons or holes have an energy distribution which is related to the energy distribution of donors or acceptors in a solution, the CTRIP spectrum contains this information as well. McIntyre and Sass demonstrated that the maximum energy of emitted photon depends on the electrode potential. This dependence is a crucial aspect of the radiative relaxation of injected electrons or holes in a metal because the energy of the emitted photon is determined by the energy of the injected electron or hole with respect to the Fermi level, which is in turn determined by the electrode potential. Ouyang and Bard applied this technique to the Pt-acetonitrile interface. ${ }^{9,10}$ Although they concluded that the relaxation was mainly to a Shockley-type surface state, a quantitative analysis of the emitted-photon spectrum was not carried out.

The difficulty in a quantitative analysis of the spectrum arises from an inadequate understanding of the radiative relaxation mechanism of the injected charge in a metal. Recently we systematically investigated the effect of the electrode metal and the redox potential of the chemical species (i.e., the energy states of electron injection) on emission properties ${ }^{11-13}$ and considered several mechanisms, including surface-plasmon excitation and decay and IP processes as the origin of photon emission at the electrochemical interface. Based on the qualitative analysis carried out, we concluded that the IP process involving the bulk band states of metal is important. ${ }^{13} \mathrm{~A}$ quantitative model to explain the details of emission process at a metal-electrolyte interface, however, is still lacking.

We also employed an experimental method to improve experimental reproducibility so that a more quantitative analysis can be made. ${ }^{14,15}$ In the electron injection procedure of McIntyre and Sass used also by Ouyang and Bard and by the present authors, the electrode potential is first pulsed to a very negative potential to generate an organic anion radical, which acts as an electron-injecting species; then it is pulsed to a positive potential so that electrons are injected from the anion radical to the metal. Usually this process is repeated many times to improve 
the $S / N$ ratio of the emitted-photon signal. The large potential pulsing may cause decomposition of the chemical species in the solution and reconstruction and roughing of the electrode surface. These effects lower the reproducibility of the photon-emission experiments. In the present technique, chemically generated solvated electrons in a hexametylphosphoric triamide (HMPA) solution are used as an electron-injecting species. Stable photon emission from the metal was observed when the potential of the metal electrode was kept relatively positive in the electrolyte solution containing solvated electrons. ${ }^{15}$ Determination of the electronic states which participate in the photon-emission process at the metalelectrolyte interface should become feasible by further quantitative analysis of the spectrum.

The information on the energy distribution of the electronic states of solvated electrons is also very important. Electrochemical generation and/or consumption processes of solvated electrons in a polar solvent have been regarded as a simple model of the heterogeneous electrontransfer process at the electrode surface. Although the kinetics of the reaction can be determined by an ordinary electrochemical method, ${ }^{16}$ the experimental evaluation of the electronic states of solvated electrons is difficult. In the photoemission study at a metal-electrolyte interface, the energy states of a quasifree electron, which is an electron in the electrolyte solution just after the emission and before relaxation, is estimated from the onset potential of photoemission. The quasifree electron then relaxes to the state known as a solvated electron. ${ }^{17}$ The emitted-photon spectrum obtained by the present technique should provide information on the electronic structure of the solvated electron and a comparison between the results of CTRIPS and that of the photoemission experiment is expected to clarify the fundamental solvation process and electrochemical reactions of solvated electrons, as our preliminary result suggests. ${ }^{14}$

In the present work, we observed photon emission from a metal electrode ( $\mathrm{Au}$ and $\mathrm{Pt}$ ) whose potential was swept or kept relatively positive in the electrolyte solution containing solvated electrons. We describe in detail the procedure used to determine the highest occupied states of solvated electrons and estimate the relaxation energy of solvated electrons in solution. A quantitative model is presented for the photon-emission process in an electrochemical system. The emitted-photon spectra are simulated by considering the transition between the bulk band states of metal while ignoring $\mathbf{k}$-vector conservation. The simulated results are compared with experiment and the validity of this model is discussed.

\section{EXPERIMENT}

Reagent-grade hexamethylphosphoric triamide (HMPA: Wako Pure Chemicals Co. Ltd.) and sodium perchlorate (Aldrich Chemicals Co.) were used as a solvent and a supporting electrolyte, respectively. They were purified and dehydrated in the usual manner. ${ }^{18}$ The electrolyte solution was prepared under a purified $\mathrm{N}_{2}$ $(99.999 \%)$ atmosphere. Solvated electrons were generat- ed by dissolving sodium metal (Wako Pure Chemicals Co. Ltd.) in HMPA solution containing $0.2 \mathrm{M} \mathrm{NaClO}_{4}$. The prepared solution showed blue color with an absorption maximum at $770 \mathrm{~nm}$, which proves the formation of solvated electrons. ${ }^{19}$ The concentration of solvated electrons was estimated to be order of the $10^{-3} \mathrm{M}$ from the weight of dissolved sodium metal. Solvated electrons generated this way could last for more than several hours at room temperature.

Metal electrodes ( $\mathrm{Au}$ and $\mathrm{Pt}$ ) were prepared on clean glass by vacuum deposition using a pure metal wire $(99.99 \%)$ as a source in $10^{-6}$ Torr vacuum. The temperature of the glass substrate during Au deposition was controlled at $300^{\circ} \mathrm{C}$ by use of a hot-plate controller (Chino Co. Ltd., DB-01-3). Pt deposition was carried out at room temperature. The deposition rate and the thickness of the film were measured with a quartz-crystal thickness monitor (ULVAC Co. Ltd., CRTM-1000). The deposition rate $(0.1 \mathrm{~nm} / \mathrm{s}$ for $\mathrm{Au}$ and $0.01 \mathrm{~nm} / \mathrm{s}$ for $\mathrm{Pt}$ ) was controlled by changing the current passed through the tungsten wire around which the metal wire was wound. The surfaces of the metal films prepared in this manner were predominantly (111) faces. ${ }^{12}$

Emission experiments were performed with a flow-type thin layer spectroelectrochemical cell made of Teflon and with an uv quartz window, combined with an external supply flask where solvated electrons were generated (Fig. 1). The separation between the uv-quartz optical window and the working electrode was set to $100 \mu \mathrm{m}$ with a Teflon spacer to minimize the absorption of emitted light by the electrolyte solution itself. The electrolyte solution was transferred from the supply flask to the thin layer compartment of the cell with a constant flow rate through a Teflon tube by applying positive $N_{2}$ pressure so that solvated electrons around the metal electrode were

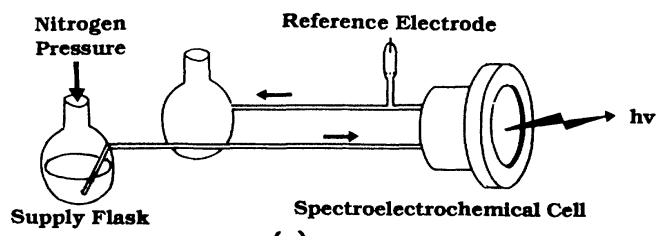

(a)

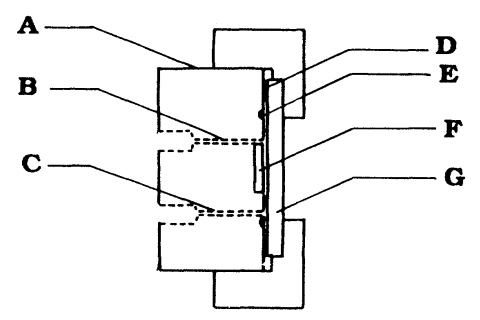

(b)

FIG. 1. Block diagram of (a) the electrolyte flow system and (b) the vertical section view of the spectroelectrochemical cell: ( $A$ ) Teflon body; $(B)$ reference electrode channel; $(C)$ solution inlet channel; $(D)$ Teflon spacer $(d=100 \mu \mathrm{m}) ;(E)$ counter electrode; $(F)$ metal working electrode; $(G)$ quartz window. 
not depleted. The potential of the working electrode was referred to a $\mathrm{Ag} /\left(0.01 \mathrm{M} \mathrm{AgNO}_{3}\right)$ reference electrode. The working electrodes potential was controlled by using a potentiostat (Nikko Keisoku, NPGS-301S). An external potential was provided by a function generator (Nikko Keisoku, NFG-03). No electrochemical reactions due to residual moisture or other impurities were detected within the potential region between 0 and $\sim-3.3 \mathrm{~V}$, where solvated electrons were generated. A positive potential limit $(0 \mathrm{~V})$ was chosen to avoid decomposition of the solvent. All the measurements were carried out at room temperature.

The emission intensity was measured with a photomultiplier tube (PMT: Hamamatsu Photonics Co. Ltd., R636) and a photon counter (NF Electronics Co. Ltd., LI-574). Spectra of emitted light from the metal electrode were obtained using an imaging spectrograph (Jobin Yvon, CP-200; $f=2.9$ ) and a multichannel detector (Hamamatsu Photonics Co. Ltd., IMD-C3330) with an image intensifier. ${ }^{20}$ The charge-coupled-device (CCD) elements were cooled to $-25^{\circ} \mathrm{C}$. In the spectrum measurements, the electrode potential was kept constant and the exposure time at the CCD system was set to $20 \mathrm{~s}$. A spectrum signal was obtained by averaging the CCD response at each potential four times. The observed photon spectrum of this system is extremely weak and has a broad shape with a full width at half maximum (FWHM) of $\sim 0.4-1.0 \mathrm{eV} .{ }^{14,15}$ To determine the peak energy of the spectrum, the noise reduction of the spectrum was carried out by Fourier transformation and inversion procedure with a quadratic filter function. ${ }^{21}$ The FWHM of the spectrum becomes broader by $\sim 0.05 \mathrm{eV}$ by this noise-reduction process. The spectrum response was calibrated using a tungsten-halogen lamp.

\section{RESULTS}

Stable photon emission from $\mathrm{Au}$ and $\mathrm{Pt}$ was observed in an electrolyte solution containing solvated electrons

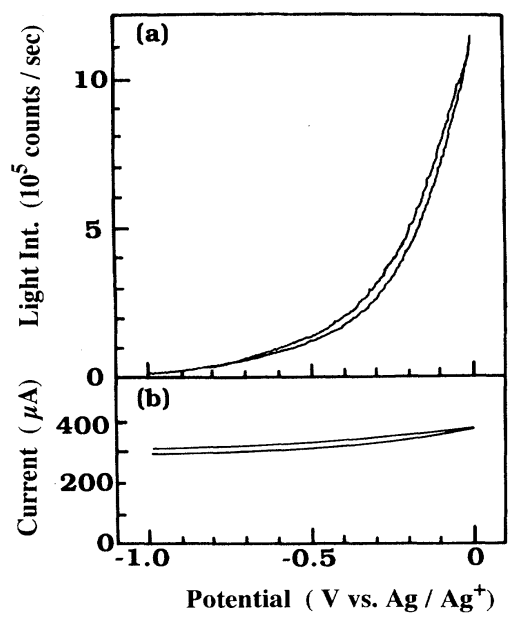

FIG. 2. Potential dependence of (a) emission intensity and (b) oxidation current at the Au electrode in $0.2 \mathrm{M} \mathrm{NaClO} 4$ HMPA solution containing solvated electrons. Scan rate: $20 \mathrm{mV} / \mathrm{s}$. when the electrode potential was relatively positive. The intensity of light emission from both metals did not change with time as long as the electrode potential was kept constant. Figure 2 shows a typical result of the potential dependence of the current and light intensity at $\mathrm{Au}$ observed by sweeping the electrode potential between -1.0 and $0 \mathrm{~V}$. Although the anodic current was almost constant, the light intensity increased significantly as the electrode potential $U_{w}$ of metal electrodes became positive. The intensity and the current were reversible for the potential scan. Similar results were obtained at a Pt electrode, although the light intensity was weaker.

Typical spectra of emitted light from $\mathrm{Au}$ electrodes while the potential was kept constant at several potential are shown in Fig. 3, without noise reduction and correction for the detector sensitivity. The shape of the spectrum became broad and the intensity increased as $U_{w}$ became positive. A similar tendency was also observed at $\mathrm{Pt}$ electrodes. Figure 4 shows the spectra at $\mathrm{Au}$ and $\mathrm{Pt}$ electrodes after noise reduction and sensitivity correction. The high-energy threshold $E_{\text {th }}$ and the peak energy $E_{p}$ of the spectra shift to high-energy direction and FWHM of the spectrum increases as $U_{w}$ becomes positive. Since the HMPA solution containing solvated electrons exhibits a broad optical-absorption band with a peak at $770 \mathrm{~nm}\left(\sim 1.6 \mathrm{eV}: \varepsilon_{770 \mathrm{~nm}} \cong 10^{4}\right),{ }^{19}$ some of the emitted photons are absorbed by the solution. It is estimated that about $20 \%$ of photons are absorbed at 770 $\mathrm{nm}$ in this system. The effect of absorption is important when the band of emitted light is close to the absorption band (between $\sim 1.4$ and $1.9 \mathrm{eV}$ ), i.e., $U_{w}$ is relatively negative. The $E_{p}$ of the corrected spectrum is located at lower energy than that of the uncorrected spectrum, e.g., by $\sim 0.05 \mathrm{eV}$ at $-0.8 \mathrm{~V}$. The determination of $E_{p}$ and the quantum efficiency was carried out using the data corrected for the effect of absorption.

Figure 5 shows the potential dependence of the quantum efficiency $\Phi$ at the Au and Pt electrode which is cal-

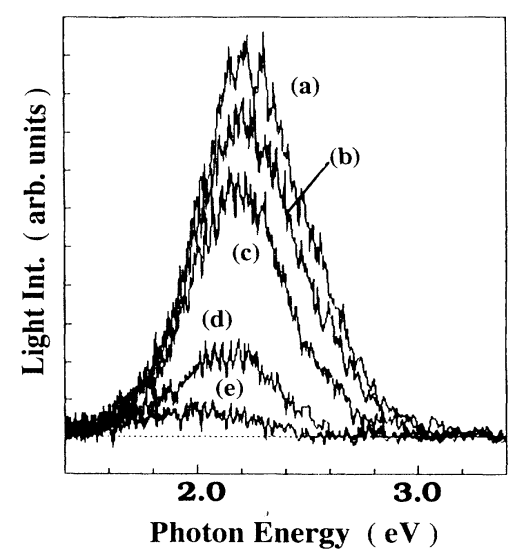

FIG. 3. Emission spectra of the Au electrode in $0.2 \mathrm{M}$ $\mathrm{NaClO}_{4} \quad \mathrm{HMPA}$ solution containing solvated electrons. The electrode potential was kept at (a) $0 \mathrm{~V}$, (b) $-0.2 \mathrm{~V}$, (c) $-0.4 \mathrm{~V}$, (d) $-0.6 \mathrm{~V}$, and (e) $-0.8 \mathrm{~V}$. No correction has been made. 


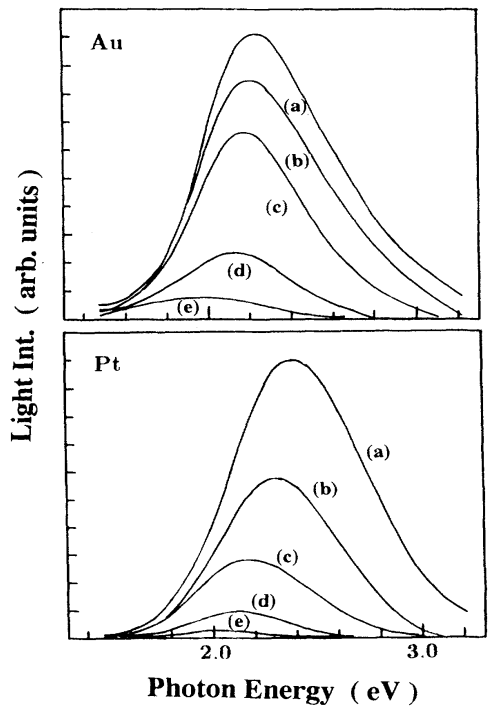

FIG. 4. Corrected spectra of the Au and Pt electrodes in 0.2 $\mathrm{M} \mathrm{NaClO}_{4}$ HMPA solution containing solvated electrons. The electrode potential was kept at (a) $0 \mathrm{~V}$, (b) $-0.2 \mathrm{~V}$, (c) $-0.4 \mathrm{~V}$, (d) $-0.6 \mathrm{~V}$, and (e) $-0.8 \mathrm{~V}$.

culated by using the anodic current and the light intensity measured by the photon-counting system and corrected for absorption by the solution. 22 The quantum efficiency is higher at more positive $U_{w}$ in both metals. The efficiency at Au electrodes is always larger than that at Pt electrodes.

The shape of the spectrum is characterized by $E_{\mathrm{th}}, E_{p}$, and the low-energy threshold $E_{l}$. Figure 6 shows the spectra in the vicinity of $E_{\mathrm{th}}$. The axis of the light intensity is magnified to show how we determined $E_{\text {th }}$. These spectra are presented without noise reduction and correction for the detector sensitivity. The values of $E_{\mathrm{th}}$ are determined somewhat arbitrarily and are shown in Fig. 6 by arrows. The energy position of $E_{l}$ was also estimated similarly, as shown in Fig. $7 .^{23}$

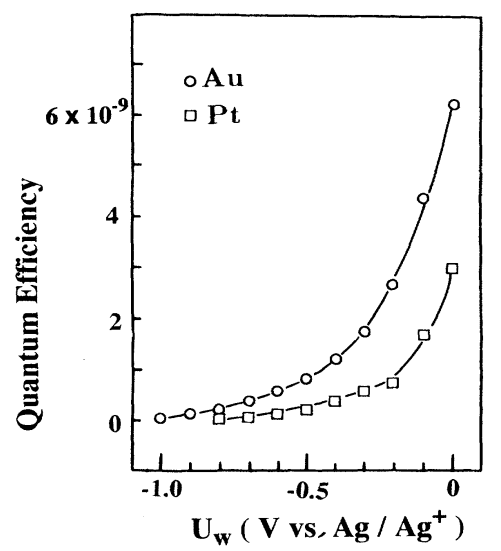

FIG. 5. Potential dependence of the quantum efficiency at the $\mathrm{Au}$ and $\mathrm{Pt}$ electrodes. Correction for the effect of the absorption by the electrolyte solution has been made.
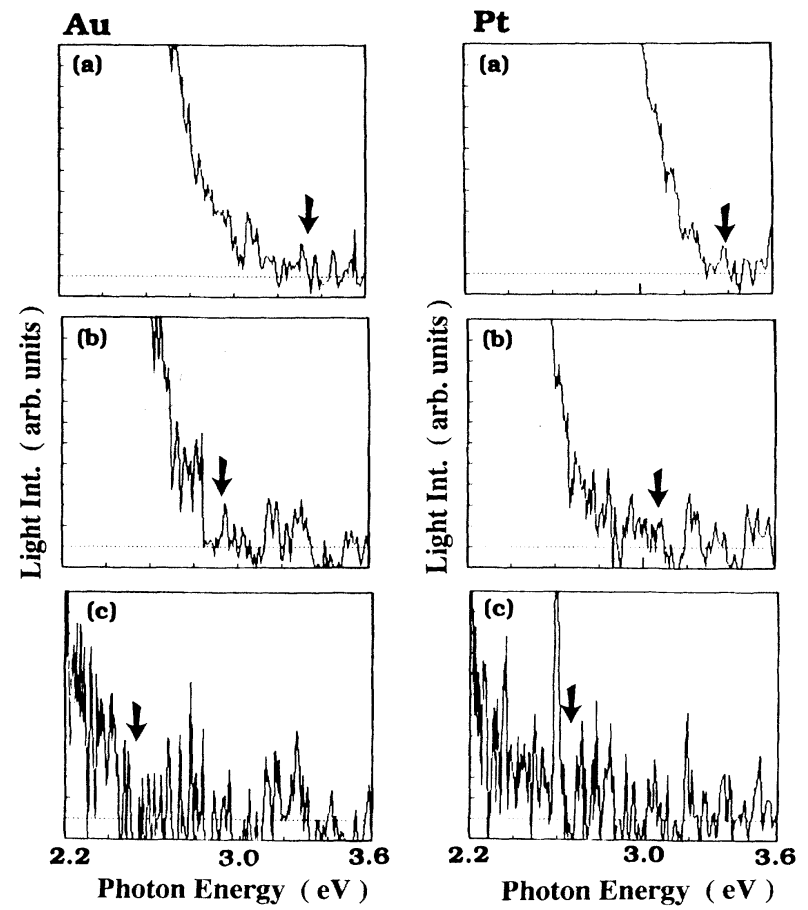

FIG. 6. Emission spectra in the vicinity of $E_{\mathrm{th}}$ 's of the Au and Pt electrodes. The electrode potential was kept at (a) $0 \mathrm{~V}$, (b) $-0.4 \mathrm{~V}$, and (c) $-0.8 \mathrm{~V}$. No correction has been made. The full scale of emission intensity at the Au electrode are enlarged by a factor of (a) 5.5 , (b) 8.3 , and (c) 17.8 with respect to the full scale of Fig. 3. At the Pt electrode, the enlargement factors are (a) 4.2, (b) 8.1, and (c) 13.8 .

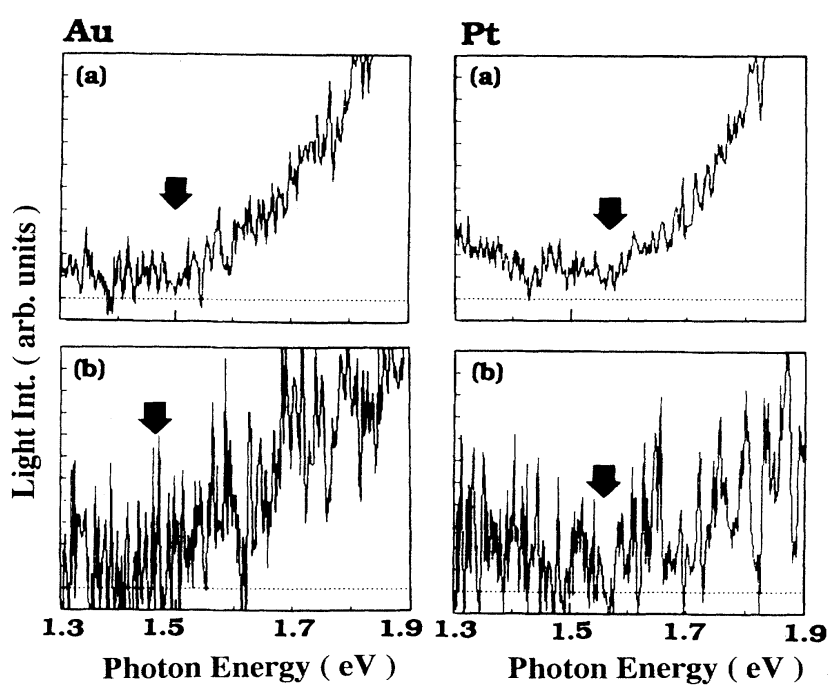

FIG. 7. Emission spectra in the vicinity of $E_{l}$ 's of the $A u$ and $\mathrm{Pt}$ electrodes. The electrode potential was kept at (a) $0 \mathrm{~V}$ and (b) $-0.8 \mathrm{~V}$. No correction has been made. The full scale of emission intensity at the Au electrode are enlarged by a factor of (a) 5.4 and (b) 17.8 with respect to the full scale of Fig. 3. At the Pt electrode, the enlargement factors are (a) 3.1 and (b) 13.8. 


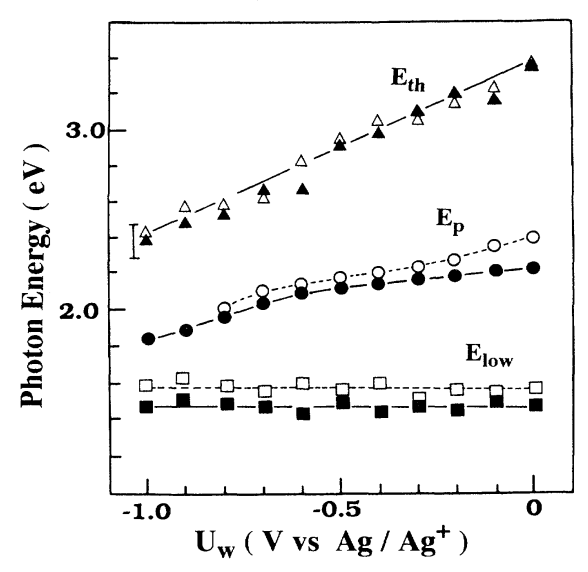

FIG. 8. Potential dependence of $E_{\mathrm{th}}, E_{p}$, and $E_{l}$ of the spectra at the $\mathrm{Au}(\Delta, \boldsymbol{O}, \mathbf{\square})$ and $\operatorname{Pt}(\triangle, \bigcirc, \square)$ electrodes.

The values of $E_{\text {th }}, E_{p}$, and $E_{l}$ determined as described above are plotted in Fig. 8 as a function of $U_{w}$. $E_{\text {th }}$ shifts in the higher-energy direction as $U_{w}$ becomes positive. The $E_{\text {th }}$ 's of the $\mathrm{Au}$ and $\mathrm{Pt}$ electrodes are almost identical and shift linearly with increasing $U_{w}$ at the rate of 1 $\mathrm{eV} / \mathrm{V}$. Although $E_{p}$ also becomes large as $U_{w}$ becomes positive, the shift of $E_{p}$ with the potential is smaller than that of $E_{\mathrm{th}}$. The difference of $E_{p}$ between the $\mathrm{Au}$ and $\mathrm{Pt}$ electrodes becomes apparent when $U_{w}$ is more positive than $-0.2 \mathrm{~V}$. The values of $E_{p}$ at the $\mathrm{Pt}$ electrode are slightly larger than that at the Au electrode in the potential region investigated here. For very negative $U_{w}$, the light intensity at the Pt electrode is very weak and it is difficult to determine $E_{p}$. Therefore, only the values obtained at more positive potentials than $-0.9 \mathrm{~V}$ were shown in Fig. 8. The energy position of $E_{l}$ depends on the metal species, but not on $U_{w}$. The $E_{l}$ at the Au electrode is located at lower energy than that at the $\mathrm{Pt}$ electrode.

\section{DISCUSSION}

\section{A. Estimation of the relaxation energy of the solvated electron}

The photon emission is thought to be due to the radiative relaxation of the injected electrons from high-energy chemical species in solution into the empty electronic states in the metal, i.e., the inverse-photoemission (IP) process. ${ }^{5-15}$ We have reported previously that $E_{\text {th }}$ of the emission spectrum at a metal-acetonitrile interface corresponds to the maximum energy of injected electrons, $E_{\text {inj }}$, which is given by the energy difference between the Fermi level in the metal and the energy level of the highest occupied electronic state of the electron-injection species in a solution. ${ }^{11-13}$ These levels can be represented by a potential scale as $U_{w}$ and $U_{\text {occ }}^{0}$, respectively, with respect to a common reference electrode. Thus, these values are correlated as $E_{\mathrm{th}} / e=E_{\mathrm{inj}} / e=-\left(U_{\mathrm{occ}}^{0}-U_{w}\right)$.

Instead of this equation, we used $E_{\mathrm{th}} / e=E_{\mathrm{inj}} / e$ $=-\left(U^{0}-U_{w}\right)$ in our previous paper, where $U^{0}$ is the redox potential of the electron-injection species. The energetic structure of the present metal-HMPA (solvated electron) interface is schematically shown in Fig. 9. From the thermodynamic definition of equilibrium, the energy level of $U^{0}$ is defined as the level where the reduced and the oxidized forms are present in equal concentrations. This condition is characterized by the equality of the densities of the occupied and unoccupied states in solution. Thus, the potential of the highest occupied electronic state of the electron-injection species, $U_{\text {occ }}^{0}$, should be located at a more negative potential than $U^{0}$, as shown in Fig. 9. The difference between the $U_{\text {occ }}^{0}$ and $U^{0}$ depends on the distribution function of these two functional forms and is usually unknown. However, the results of photon-emission experiment at a metal-acetonitrile-solution interface suggests that $U_{\mathrm{occ}}^{0}$ can be approximated by $U^{0}$, i.e., the energy difference between $U^{0}$ and $U_{\text {occ }}^{0}$ should be small compared with the experimental precision of this system.

By applying this relation to the present system, $U^{0}$ or $U_{\text {occ }}^{0}$ of the solvated electron is estimated from $E_{\text {th }}$ and $U_{w}$ (Fig. 8) as $-3.4 \pm 0.1 \mathrm{~V}$ vs $\mathrm{Ag} / \mathrm{Ag}^{+}$. Although the various conditions in this system such as the concentration of solvated electrons, temperature, and the resolution of the optical system should be controlled or improved for the more accurate determination of $E_{\mathrm{th}}$, this value is comparable with the standard electrode potential of the solvated electron determined electrochemically at the $\mathrm{Pt}$ electrode in HMPA solution containing $0.2 \mathrm{M}$ $\mathrm{NaClO}_{4}$ at $5{ }^{\circ} \mathrm{C}\left[-3.44 \mathrm{~V}\right.$ vs $\left.\mathrm{Ag} /\left(0.1 \mathrm{M} \mathrm{AgClO}_{4}\right)\right]$. ${ }^{16}$

Solvated electrons can be generated by photon radiation to electrodes in polar solvents. From the onset potential of photocurrent and incident photon energy, one can identify the energy level of an electron injected from the metal electrode into solution. This energy corresponds to the level of a quasifree electron, $U_{q}$ (Fig. 9). $U_{q}$ was estimated to be $-3.82 \mathrm{~V}$ in $0.2 \mathrm{M} \mathrm{NaClO}_{4}$ in the HMPA system. ${ }^{17}$ The difference between $U_{q}$ and the value obtained in this study corresponds to the relaxation energy of solvated electron in the HMPA solution and is determined to be $\sim 0.4 \mathrm{eV}$.

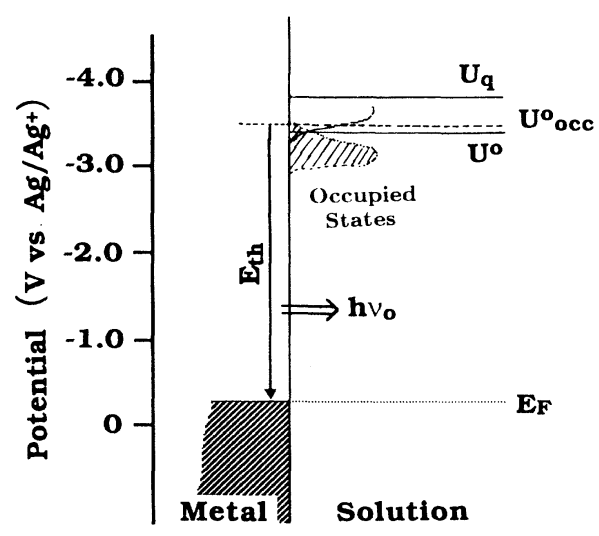

FIG. 9. The energetic structure of the metal-HMPA-solution (solvated electron) interface. The value of $U_{q}$ is of Ref. 17. 
The present result proves that photon-emission measurement in the electrochemical system is useful for the in situ determination of $U^{0}$ or $U_{\text {occ }}^{0}$ of the electrochemical species in solution. This technique also may be applicable for unstable and/or irreversible systems where the determination of $U^{0}$ by ordinary electrochemical measurement is impossible.

\section{B. Model for photon emission at a metal-enelectrolyte interface}

Several mechanisms for photon emission at a metal-electrolyte-solution interface have been proposed by several groups. ${ }^{5-10}$ We have proposed that the IP process involving the bulk band states of metal is dominant in this phenomenon. ${ }^{13,15}$ There is, however, no quantitative model which can explain the characteristics of the emitted photons. In this section we present a quantitative model for the photon-emission process at an electrochemical system assuming that the photon emission is solely due to the radiative transition of energetic electrons between the bulk band states of metal. $\mathbf{k}$-vector conservation is ignored in this treatment. The simulated spectra are derived from the model and are compared with experiment.

\section{Qualitative description of the present model for the photon-emission process}

The present model for photon-emission process at an electrochemical interface is based on an established model for the IP process at IPS in vacuum. ${ }^{24,25}$ The specificity of the present emission process is in the electron-injection process via an electrochemical ET reaction. The feature of the injected electrons in the electrochemical system should be entirely different from that in IPS. While the injected electrons have relatively high monochromatic energy (typically 9-30 eV) in IPS, the injected electrons of the present system have energy of a few eV above the Fermi level and have a certain energy distribution. Thus, the model of the IP process should be modified before application to the present electrochemical system.

In the model for IPS, the IP process is usually divided into the following three steps. ${ }^{26}$ The first step is an electron injecting process in which the incoming electron couples into one of the unoccupied states of the solid. The second step is a scattering process of injected electrons in metal. Most of the injected electrons with energy of a few tens of $\mathrm{eV}$ above the Fermi level will decay nonradiatively via electron-electron inelastic scattering process in the metal. This is the physical origin of the very short mean free path $(\sim 1 \mathrm{~nm})$ of electrons in this energy region. A certain fraction of injected electrons decay radiatively to unoccupied states at lower energy in the metal, yielding photons. This photon radiation process is considered to be the third step.

In an electrochemical system, the relatively low energy of the injected electrons system leads to a low probability for inelastic scattering. The injected electrons are expected to penetrate into the metal by a distance of the order of the mean free path, e.g., a few tens of nm. ${ }^{27}$ Thus, radiative relaxation of the energetic electron occurs within a certain depth. Since the emitted photons inside the metal should be partially absorbed by the metal before they arrive at the metal surface, a photon-absorption step by the metal should be added to the model as the fourth step in this case.

Based on the above consideration, the photon-emission process at the electrochemical system is divided into four steps: the electron-injection, electron-scattering, photonemission, and photon-absorption steps. Each step of the photon-emission process at the electrochemical system may be described qualitatively as follows. The first step is the electron-injection step. Energetic electrons are injected from the electrolyte solution into the metal electrode via electrochemical ET reactions. The energy distribution of the injected electrons in the metal reflects the energy distribution of the occupied states of the electron donor in the electrolyte solution and the unoccupied states of the metal. The second step is the scattering process of injected electrons in the metal. Injected electrons with energy of a few eV above the Fermi level are scattered via various processes, such as electron-defect, electron-impurity, electron-phonon, and electron-electron scattering. ${ }^{27}$ Among these scattering processes, the most important inelastic collision processes are electronphonon and electron-electron scattering processes which create electron-hole pairs. ${ }^{24,25,28,29}$ Although the probability of electron-phonon scattering is almost comparable to, or larger than, that of electron-electron scattering in the energy region of a few eV, ${ }^{27,30}$ the maximum energy loss associated with electron-phonon scattering is much smaller ( $\leq 20 \mathrm{meV}$ ) (Ref. 31) than that of electronelectron scattering. In the present model, the energy loss due to electron-phonon scattering is, therefore, ignored. The mean free path is determined by using the probability of the electron-electron scattering. The values of the mean free paths in $\mathrm{Au}$ have been estimated as $\sim 50 \mathrm{~nm}$ for the $2.0-\mathrm{eV}$ electron and $\sim 20 \mathrm{~nm}$ for the $3.0-\mathrm{eV}$ electron. ${ }^{30}$ The values in $\mathrm{Pt}$ are estimated as $\sim 13 \mathrm{~nm}$ for the $2.0-\mathrm{eV}$ electron and $\sim 8 \mathrm{~nm}$ for the $3.0-\mathrm{eV}$ electron. ${ }^{27}$ The third step is the photon-emission process. It is assumed that radiative transition takes place between bulk band states of the metal. $\mathbf{k}$-vector conservation is ignored in the transition. This assumption is the most important basis to explain the experimental results. The fourth step is the photon-absorption step. The escape depth of the emitted photon of visible wavelength, which can be calculated from the inverse of the optical-absorption constant of the metal, is around $10-20 \mathrm{~nm} .{ }^{32}$ This value is comparable to the mean free path of the electron. A certain fraction of the photons which are generated inside the metal is absorbed before they arrive at the metal surface. The fraction of the absorbed photons are determined by the depths where the photons are generated and the optical-absorption constant of the metal.

\section{Formulation of the model and simulation of the spectra}

In this section, each step of the four-step model for the photon-emission process at an electrochemical interface is described quantitatively and is formulated and, finally, the intensity of the emitted photons are calculated as a 
function of the photon energy to simulate the spectra.

a. Electron-injection process. Energetic electrons are injected into the semi-infinite metal via an electrochemical ET reaction. Using the concept that electron transfer can take place only between electronic states of equal energies, one being occupied and the other unoccupied, the number of injected electrons per second with energy between $E$ and $E+d E, I_{0}(E) d E$, can be approximated as

$$
I_{0}(E) d E \propto \rho_{m}(E)\{1-f(E)\} T(E) N_{s}(E) d E,
$$

where $\rho_{m}(E)$ and $N_{s}(E)$ are the density of the electronic states in the metal and the number of occupied electronic states in the solution at energy $E$, respectively. ${ }^{33}$ The energy is given with respect to the bottom of the conduction band of the metal. Here $f(E)$ is the Fermi distribution function and $T(E)$ is the tunneling probability at energy $E$. In the present calculation, it is assumed that $T(E)$ is independent of $E$. Thus, the number of injected electrons per second is proportional to the product of $\rho_{m}(E)\{1-f(E)\}$ and $N_{s}(E)$. The direction of impinging electrons is assumed to be perpendicular to a metal surface. The values of $\rho_{m}(E)$ for $\mathrm{Au}$ and $\mathrm{Pt}$ are taken from the results calculated by Smith and co-workers and are plotted in Fig. 10 as a function of energy. ${ }^{34,35}$

Although the determination of the energy distributions of electrons in solution, $N_{s}(E)$, is still a very controversial issue at present, the distribution used in this calculation is assumed as follows. One of the acceptable models for determining the distribution is the vibration-rotation interaction model, ${ }^{36}$ in which $N_{s}(E)$ is defined as the distribution of the energy required for the transition from the reduced state to the oxidized state in a solution. The present electron-transfer-reaction process involves the states of the solvated electron as the reduced form and the empty trap which consists of highly oriented solvent molecules as the oxidized form. It is generally believed that optical absorption by the solution containing the sol-

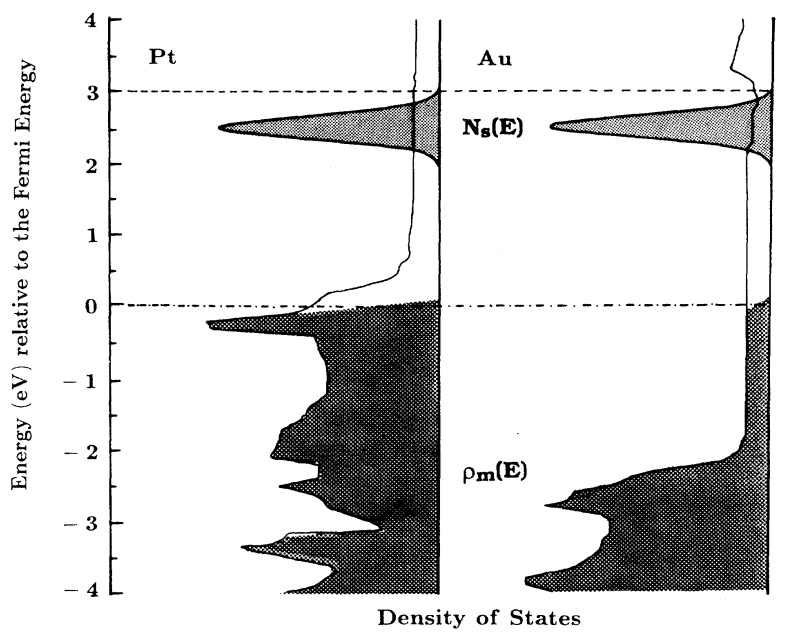

FIG. 10. Density of the electronic states of metal, $\rho_{m}(E)$, and the solution, $N_{s}(E)$. The values of $\rho_{m}(E)$ were taken from Ref. 35. The zero energy corresponds to the Fermi level. vated electron is due to the removal of the electron from the solvation trap to the outside trap. Henglein estimated the width of the distribution of the transition energy between the two states from the width of the absorption band. ${ }^{37} \mathrm{~A}$ similar approach is taken in this process in order to obtain information about $N_{s}(E)$ crudely from the shape of the absorption band. From the fact that the absorption band at $770 \mathrm{~nm}$ has a FWHM of $0.5 \mathrm{eV}$, the width of $N_{s}(E)$ is roughly estimated as $\sim 0.5 \mathrm{eV}$. The Gaussian distribution of the states with a width of $0.5 \mathrm{eV}$ is assumed in this calculation for simplicity. ${ }^{38}$ The energy dependence of $N_{s}(E)$ is also shown in Fig. 10.

b. Effect of inelastic scattering on the energy of injected electrons. The energy of injected electrons changes via the electron-electron inelastic scattering process as propagating in the metal bulk. The energy distribution of the injected electrons at depth $x$ is calculated from the probability that the electron with the initial energy $E$ is scattered to a final state with energy $E-\Delta E, N(E, \Delta E)$, and the fraction of electrons with energy $E$ which arrive at depth $x$ without scattering, $g(E, x)$.

In electron-electron scattering processes, an electron with initial energy $E$ is scattered to a final state with energy $E-\Delta E$, creating an electron-hole pair by raising an electron from a level of energy $E^{\prime}$ which is below the Fermi level to a level $E^{\prime}+\Delta E$ which is above the Fermi level (Fig. 11). The function $N(E, \Delta E)$ was calculated by Berglund and Spicer ${ }^{29}$ using the approximation which assumed that the matrix element of the transition is independent of the $\mathbf{k}$ vectors of the electrons involved. Using this approximation, the number of the scattering events leading to an energy loss of $\Delta E, n(E, \Delta E)$ is determined by the density of the states involved, i.e., $\rho_{m}(E-\Delta E), \rho_{m}\left(E^{\prime}\right)$, and $\rho_{m}\left(E^{\prime}+\Delta E\right)$, and is given by

$$
\begin{aligned}
n(E, \Delta E)=\int_{0}^{E} & \rho_{m}(E-\Delta E)\{1-f(E-\Delta E)\} \rho_{m}\left(E^{\prime}\right) f\left(E^{\prime}\right) \\
& \times \rho_{m}\left(E^{\prime}+\Delta E\right)\left\{1-f\left(E^{\prime}+\Delta E\right)\right\} d E^{\prime} .
\end{aligned}
$$

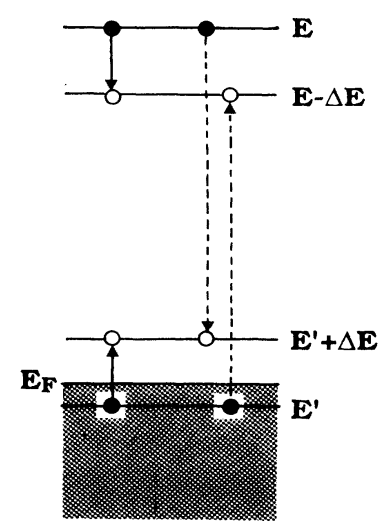

FIG. 11. Energy-level diagram for the electron-hole pairproduction process. Two possible scattering processes, indicated by solid and dashed lines, involve the same four electronic states. 
The total number of the transition events for the excited states, $n_{t}(E)$, is obtained by the integration over all energetically accessible states, i.e.,

$$
n_{t}(E)=\int_{0}^{E} n(E, \Delta E) d(\Delta E)
$$

Thus, the normalized probability, $N(E, \Delta E)$, is given by

$$
N(E, \Delta E)=\frac{2 n(E, \Delta E)}{n_{t}(E)} .
$$

The electron-hole pair can be produced via two different mechanisms leading to the same final state as shown in Fig. 11 by solid and dotted lines. The same four electronic states are involved in the two processes which have equal transition probability. Thus, this function is normalized to two equivalent events in Eq. (4).

$g(E, x)$ is determined by the mean free path $l_{e}(E)$ for the scattering process and the traveling length of the energetic electrons in metal. The mean free paths for electron-electron scattering are calculated as a function of the electron energy. ${ }^{27,30}$ The values of the calculated mean free path are used for the determination of $g(E, x)$. The traveling length is estimated by using the following assumption. Although the normal incidence of the electron injection is assumed in the model, the electrons should be deflected at the metal surface and have a certain distribution of propagation angle $\theta$ with respect to the surface normal. ${ }^{39}$ The traveling length of the deflected electron at a certain depth $x$ is determined by $\theta$ and is given by $x / \cos \theta$. The traveling length of a deflected electron is longer than that of an undeflected electron. The function of $g(E, x)$ is determined by Kanter, assuming the scattering distribution of the normal component is $\cos \theta$ and is given by ${ }^{40}$

$$
g(E, x)=\left[1-\zeta\left\{1-\zeta+\zeta^{2} G(\zeta)\right\}\right] \exp (-\zeta),
$$

where $\zeta=x / l_{e}(E)$ and $G(\zeta)$ is the incomplete $\Gamma$ function. Equation (5) gives that $g(E, x)$ decreases with $x$ faster than a simple exponential function.

c. Photon-emission process. The number of the radiative relaxation events per unit time which emit the mono- chromatic photon with energy $h v_{0}$ via the spontaneous emission process is given by ${ }^{41}$

$$
A\left(h v_{0}\right)=a \frac{64 \pi^{4} v_{0}^{3}}{3 h c^{3}}
$$

where the factor $a$ is an arbitrary constant including the square of the matrix element and is assumed to be independent of electron energy in this model. The number of the radiative relaxation events per unit electron propagation length is represented by $A\left(h v_{0}\right) / v_{g}(E) . v_{g}(E)$ is the velocity of an electron with energy $E$ in metal and is given by

$$
v_{g}(E)=\left(\frac{2}{m} E\right)^{1 / 2} .
$$

The number of the radiative transition events in which the photon with energy $h v_{0}$ is emitted between $x$ and $x+d x$ from the surface, $\eta\left(E, h v_{0}, x\right) d x$, is given by

$$
\eta\left(E, h v_{0}, x\right) d x=\frac{A\left(h v_{0}\right)}{v_{g}(E)} \exp \left[-\frac{A\left(h v_{0}\right)}{v_{g}(E)} x\right] d x .
$$

Although the absolute value of $A\left(h v_{0}\right)$ is not available at present, $A\left(h v_{0}\right)$ should be lower than the nonradiative relaxation rate, which is estimated on the order of $10^{15}$ $\mathrm{s}^{-1}$, by several orders of magnitude. ${ }^{42}$ The value of $x / v_{g}(E)$ considered here is $\sim 10^{-16}-10^{-15} \mathrm{~s}$ because $x$ and $v_{g}(E)$ are on the order of $10 \mathrm{~nm}$ and $10^{6} \mathrm{~m} / \mathrm{s},{ }^{31}$ respectively. Thus, the $\exp \left\{-\left[A\left(h v_{0}\right) / v_{g}(E)\right] x\right\}$ is approximated by unity in the present calculation. Equation (8) shows that the probability of photon radiation is proportional to the third power of the photon energy and to the inverse square of the electron energy.

The probability of photon radiation with energy $h v_{0}$ induced by electrons with energy $E$ between $x$ and $x+d x$ from the surface, $P\left(E, h v_{0}, x\right) d x$, is proportional to the product of $\eta\left(E, h v_{0}, x\right) d x$ and the density of the states to which the injected electrons relax radiatively, and is given by

$$
\begin{aligned}
P\left(E, h v_{0}, x\right) d x \propto[ & g(E, x) \eta\left(E, h v_{0}, x\right) \rho_{m}\left(E-h v_{0}\right)\left\{1-f\left(E-h v_{0}\right)\right\} \\
& +\{1-g(E, x)\} \int_{0}^{E-h v_{0}} \eta\left(E-\Delta E, h v_{0}, x\right) \rho_{m}\left(E-\Delta E-h v_{0}\right) \\
& \left.\times\left\{1-f\left(E-\Delta E-h v_{0}\right)\right\} N(E, \Delta E) d(\Delta E)\right] d x .
\end{aligned}
$$

The first term in Eq. (9) represents the primary radiative transition event to unoccupied states of metal. The second term represents the contribution of the electron scattered once to the radiative signal. These processes are shown schematically in Fig. 12. Only photons which are generated on the order of the escape depth of the photon can arrive at the metal surface. Since the escape depth of the emitted photon of visible wavelength is $\sim 10-20 \mathrm{~nm}$ at $\mathrm{Au}$ and $\mathrm{Pt}$ electrodes, as mentioned before, and $l_{e}(E)$ is almost equal to or larger than the escape depth of the photon, the contribution of electrons scattered more than twice to the photon-emission process can be ignored. Thus, in the present model, only the effect of single scattering is considered. 

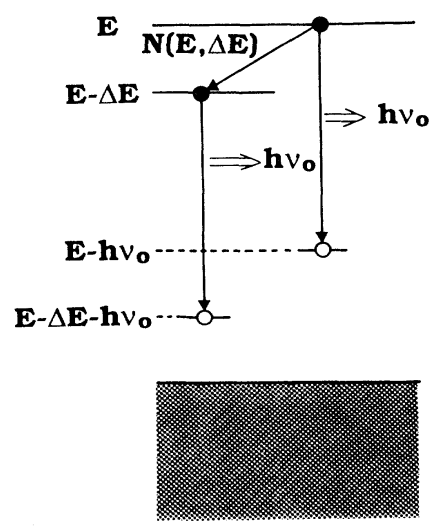

FIG. 12. Energy-level diagram for photon emission preceding or following the energy-loss process.

d. Fraction of photons which can escape into solution. A photon generated at depth $x$ and traveling at an angle $\theta$ with respect to the surface normal must travel a distance $x / \cos \theta$ before reaching the surface. The fraction of photons which are generated at $x$ and reach the surface, $Q$, is given by

$$
Q=\exp \left[-\frac{\alpha\left(h v_{0}\right) x}{\cos \theta}\right)
$$

where $\alpha\left(h v_{0}\right)$ is the optical-absorption constant of the photon with energy $h v_{0}$. The solid angle between $\theta$ and $\theta+d \theta$ is given by $2 \pi \sin \theta d \theta .{ }^{43}$ The fraction of photons in this solid angle is determined to be $\left(\frac{1}{2}\right) \sin \theta d \theta$. Thus, the total fraction of photons with energy $h v_{0}$ generated at depth $x$ which escape into the solution without absorption by metal, $F\left(h v_{0}, x\right)$, is given by

$$
F\left(h v_{0}, x\right)=\frac{1}{2} \int_{0}^{\pi / 2} \sin \theta \exp \left[-\frac{\alpha\left(h v_{0}\right) x}{\cos \theta}\right) d \theta .
$$

Equation (11) shows that $F\left(h v_{0}, x\right)$ decreases with $x$ much faster than a simple exponential function.

e. Calculation of emission intensity as a function of photon energy. The intensity of the emitted photons with energy $h v_{0}$, which are induced by the injected electron with energy between $E$ and $E+d E$ between $x$ and $x+d x$ from the surface, is given by the product of $I_{0}(E) d E$, $P\left(E, h v_{0}, x\right) d x$, and $F\left(h v_{0}, x\right)$. Summing up the contributions of all possible values of $x$ and $E$, we obtain the relative intensity of photon radiation with energy $h v_{0}$ from the metal surface, $I_{\text {INT }}\left(E_{\text {inj }}, h v_{0}\right)$, induced by electrontransfer reactions in a solution.

$$
\begin{aligned}
I_{\mathrm{INT}}\left(E_{\mathrm{inj}}, h v_{0}\right) \propto \int_{0}^{E_{\mathrm{inj}}} \int_{0}^{\infty} & I_{0}(E) P\left(E, h v_{0}, x\right) \\
& \times F\left(h v_{0}, x\right) d x d E
\end{aligned}
$$

Simulated spectra are obtained from the calculation of the relative intensity of the emitted photon in the energy region between 1.5 and $3.5 \mathrm{eV}$ at a given $E_{\text {inj }}$ using Eq. (12). Figure 13 shows typical simulated spectra. Since the matrix elements are treated as an arbitrary constant, the absolute intensity of $\mathrm{Au}$ and $\mathrm{Pt}$ cannot be compared in this calculation. Thus, the spectra obtained for $E_{\text {inj }}=3.4 \mathrm{eV}$ at $\mathrm{Au}$ and $\mathrm{Pt}$ are normalized at the peak in Fig. 13(a). For both metals, broad and structureless spectra were obtained. The contribution of the electron scattered once to the emitted photon intensity is also shown in Fig. 13(a). The emitted-photon intensity due to the primary radiative transition event comprises $97 \%$ and $69 \%$ of the whole radiation intensity in the range from 1.5 to $3.5 \mathrm{eV}$ at $\mathrm{Au}$ and $\mathrm{Pt}$, respectively. The photon emission due to the scattered electron contributes more at the relatively low-energy region of the spectra. The $E_{\text {inj }}$ dependences at $\mathrm{Au}$ and $\mathrm{Pt}$ are shown in Figs. 13(b) and $13(\mathrm{c})$, respectively. The calculated emitted-photon intensity increases as $E_{\text {inj }}$ becomes large, although that of the relatively lower-energy photon is not so much. It is clear that the relative intensity of high-energy photons at $\mathrm{Pt}$ is stronger than that at $\mathrm{Au}$. The $E_{p}$ of $\mathrm{Pt}$ is located at a slightly higher-energy position than that of Au. The $E_{l}$ of the spectra cannot be determined from the spectra.

\section{Comparison between the experimental and calculated results}

The simulated spectra reproduce most of the important aspects of the experimental results. One of the important
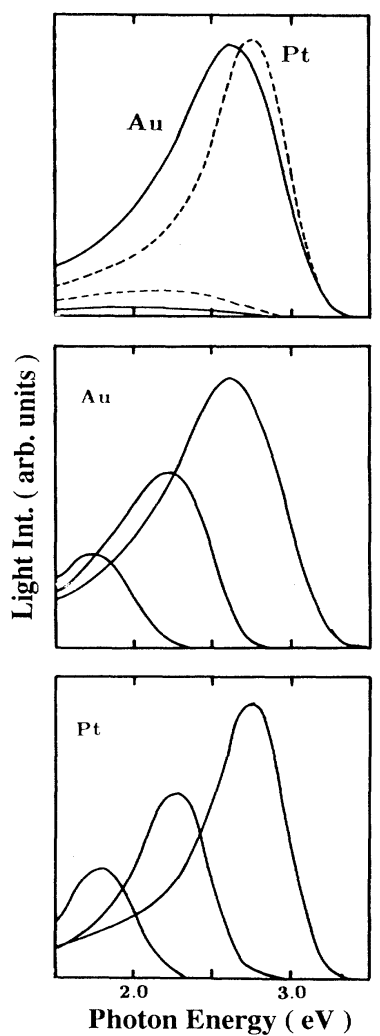

FIG. 13. Simulated spectra of the Au and Pt electrodes. In (a), $E_{\mathrm{inj}}$ is $3.4 \mathrm{eV}$. The emission due to the electrons scattering once is shown as fine solid and dotted lines. In (b) and (c), $E_{\mathrm{inj}}$ is $3.4,2.7$, and $2.2 \mathrm{eV}$. 
experimental features of the observed spectrum at a metal-electrolyte interface is the $E_{\text {inj }}$ dependence of the spectrum. As $E_{\text {inj }}$ increases, i.e., $U_{w}$ becomes positive, $E_{\text {th }}$ increases linearly by $1 \mathrm{eV} / \mathrm{V}$ and $\Phi$ increases significantly. Figures 13(b) and 13(c) show the linear shift of $E_{\mathrm{th}}$ and the increase of the emission intensity as $E_{\mathrm{inj}}$ increases. The linear dependence of $E_{\text {th }}$ on $U_{w}$ can be readily explained by the IP process. The $E_{\text {inj }}$ dependence of $\Phi$ is reproduced as well by the simulation in which the intensity of the emission is assumed to depend not only on the density of the unoccupied states of metal, $\rho_{m}(E)\{1-f(E)\}$, but also on the transition probability of the radiative event, $\eta$, which is a function of the emitted photon energy, $h v_{0}$. The $E_{\text {inj }}$ dependence of $\Phi$ seems to reflect that of $\eta$. Another important experimental feature is the metal dependence of the spectrum. The values of $E_{p}$ and $E_{l}$ at $\mathrm{Pt}$ are slightly larger than those at Au (Fig. 7). Although $E_{l}$ cannot be determined by the simulated spectra, larger contribution of higher-energy photons at $\mathrm{Pt}$ is reproduced by the simulation, as shown in Fig. 13(a). The metal dependence of the spectrum seems to be explained by the difference of the bulk band structure of metal. It is shown that the localized highdensity $d$ band just above the Fermi level in Pt results in the stronger emission of higher-energy photons compared with that of $\mathrm{Au}$, which has a relatively low-density $s p$ band with flat structure in the vicinity of the Fermi level.

Thus, we conclude that the agreement between experiment and our calculated results is fair and the contribution of the bulk band states of metal to IP processes is very important for photon emission in an electrochemical system.

There are, however, some discrepancies between experiment and the simulated results. The simulation did not reproduce the lower-energy part of the spectra as well as the $E_{\text {inj }}$ dependence of $E_{p}$. In the present model only the bulk properties of the system are considered. Although the effect of the interface on the relaxation process at metal is not clear at present, the contribution of this effect should be important and must be taken into account. There are other possible explanations for the discrepancy. In the present model the conservation of crystal momentum, $\mathbf{k}$, in the electronic transition is not assumed. If the direct transition at a certain point in the Brillouin zone, in which the matrix elements have relatively large value compared with that in other transitions, occurs strongly, ${ }^{44}$ the emitted-photon spectra should be affected by such a transition, which emits the fixedenergy photon. Thus, the identification of $\mathbf{k}$ space in the transition seems to be very important. Furthermore, in the present simulation, the electron-transfer process at the metal-electrolyte interface is extremely simplified. The shape of the spectrum largely depends on the energy distribution of the injected electron as well as the density of the states of metal. Thus, the electron-transfer process at an electrochemical interface and $N_{s}(E)$ should be treated more rigorously. Another possible contribution is the plasmon excitation-decay process, which can be induced by electron injection into the metal surface. The coupling of the plasmon with the incoming energetic electrons has been considered in the resonant IPS process $^{45,46}$ and the photon-emission process in tunneling junction systems. ${ }^{47}$ Because of the similarity of energies involved, these effects are considered as possible contributions to the emission process. ${ }^{13,15}$ More quantitative treatment of these contributions may improve the model for the photon-emission process at the metal-electrolyte interface.

\section{CONCLUSION}

Photon emission at a metal-electrolyte-solution interface was observed using a solvated electron as the electron-injection species. Stable photon emission was observed in this system compared with the previous method, in which the electron-injecting species is generated by pulsing electrode potential. The reproducibility of the experiment was also improved. It was proved that $U_{\text {occ }}^{0}$ or $U^{0}$ of the solvated electron can be determined via spectrum measurement. This technique should be applicable to another system where the determination of $U^{0}$ is impossible due to the instability and irreversibility of the redox system.

The quantitative model for the photon-emission process at an electrochemical interface is presented. In this model, it is assumed that the radiative transition takes place between the bulk band states of metal, ignoring the conservation of the $\mathbf{k}$ vector. The emission processes are divided into four steps: electron injection, electron scattering, photon emission, and photon absorption. Each step was formulated and the simulated spectra were derived. Several characteristics of the emission spectra were reproduced by the simulated spectra. These similarities support the validity of the present model for the photon emission. There are, however, some discrepancies between the experimentally observed spectra and simulated ones. For the complete explanation of the emission process at an electrochemical interface, we should treat the scattering process of injected electron in metal more precisely and improve the understanding of the radiative transition process of the energetic electron in metal. The study of photon-emission phenomenon at a metal-electrolyte-solution interface should provide a new insight into the role of electronic states on the ET reaction at an electrochemical interface.

\section{ACKNOWLEDGMENT}

This work was supported by Grants-in-Aid for Scientific Research of the Ministry of Education, Science and Culture, Japan (Nos. 02453001 and 04555191).
${ }^{*}$ To whom all correspondence should be addressed.

${ }^{1}$ R. Sonnenfeld, J. Schneir, and P. K. Hansma, in Modern Aspects of Electrochemistry, edited by R. E. White, J. O'M. Bockris, and B. E. Conway (Plenum, New York, 1990), Vol.
21, p. 1 .

${ }^{2}$ B. Beden and C. Lamy, in Spectroelectrochemistry, edited by J. Gale (Plenum, New York, 1988), Chap. 5.

${ }^{3}$ J. B. Pendry, Surf. Sci. 57, 679 (1976). 
${ }^{4}$ N. V. Smith and D. P. Woodruff, Prog. Surf. Sci. 21, 295 (1986).

${ }^{5}$ R. McIntyre and J. K. Sass, J. Electroanal. Chem. 196, 199 (1985).

${ }^{6}$ R. McIntyre and J. K. Sass, Phys. Rev. Lett. 56, 651 (1986).

${ }^{7}$ R. McIntyre, D. K. Roe, J. K. Sass, and W. Storck, J. Electroanal. Chem. 228, 293 (1987).

${ }^{8}$ R. McIntyre, D. K. Roe, J. K. Sass, and H. Gerischer, in Electrochemical Surface Science, ACS Symposium Series Vol. 378, edited by M. P. Soriaga (American Chemical Society, Washington, DC, 1988), p. 233.

${ }^{9}$ J. Ouyang and A. J. Bard, J. Phys. Chem. 91, 4058 (1987).

${ }^{10}$ J. Ouyang and A. J. Bard, J. Phys. Chem. 92, 5201 (1988).

${ }^{11}$ K. Uosaki, K. Murakoshi, and H. Kita, Chem. Lett. 1990, 1159.

${ }^{12}$ K. Uosaki, K. Murakoshi, and H. Kita, J. Phys. Chem. 95, 779 (1991).

${ }^{13}$ K. Murakoshi and K. Uosaki, J. Phys. Chem. 96, 4593 (1992).

${ }^{14} \mathrm{~K}$. Murakoshi and K. Uosaki, J. Electroanal. Chem. 308, 351 (1991).

${ }^{15}$ K. Murakoshi and K. Uosaki, J. Vac. Sci. Technol. A 10, 2981 (1992).

${ }^{16}$ Y. Kanzaki and S. Aoyagui, J. Electroanal. Chem. 36, 297 (1972).

${ }^{17}$ K. Yamashita and H. Imai, Bull. Chem. Soc. Jpn. 50, 1066 (1977).

${ }^{18}$ D. D. Perrin, W. L. F. Armarego, and D. R. Perrin, Purification of Laboratory Chemicals, 2nd ed. (Pergamon, New York, 1981).

${ }^{19}$ M. Brooks and R. R. Dewald, J. Phys. Chem. 72, 2655 (1968).

${ }^{20} \mathrm{This}$ system covers the photon-energy range $1.4-3.5 \mathrm{eV}$. The resolution was $\sim 4 \mathrm{~nm}$.

${ }^{21}$ E. E. Aubarel, J. C. Myland, K. B. Oldham, and C. G. Zoski, J. Electroanal. Chem. 184, 239 (1985).

${ }^{22}$ The quantum efficiency of this process is estimated by assuming that $\sim 90 \%$ of photons are transmitted through the quartz window, and that the counting efficiency of the PMT is $\sim 10 \%$.

${ }^{23} \mathrm{The} \mathrm{CCD}$ responses at the energy region below $1.4 \mathrm{eV}$ are due to secondary diffraction of the incident light.

${ }^{24}$ V. Dose, Surf. Sci. Rep. 5, 337 (1985).

${ }^{25}$ V. Dose, and G. Reusing, Appl. Phys. 23, 131 (1980).

${ }^{26}$ D. P. Woodruff, N. V. Smith, P. D. Johnson, and W. A. Royer, Phys. Rev. B 26, 2943 (1982).
${ }^{27}$ C. R. Crowell, and S. M. Sze, in Physics in Thin Films, edited by G. Hass and R. F. Thun (Academic, New York, 1967), Vol. 4, p. 325.

${ }^{28}$ B. Lesiak, A. Jablonski, Z. Prussak, and P. Mrozek, Surf. Sci. 222, 213 (1989).

${ }^{29}$ C. N. Berglund and W. E. Spicer, Phys. Rev. 136, A1030 (1964); 136, A1044 (1964).

${ }^{30}$ W. F. Krolikowski and W. E. Spicer, Phys. Rev. B 1, 478 (1970).

${ }^{31}$ C. Kittel, Introduction to Solid State Physics, 6th ed. (Wiley, New York, 1986), Chap. 2.

${ }^{32}$ D. M. Kolb, in Spectroelectrochemistry, edited by J. Gale (Plenum, New York, 1988), Chap. 4.

${ }^{33}$ J. O'M. Bockris and S. U. M. Khan, Quantum Electrochemistry (Plenum, New York, 1979), Chap. 8.

${ }^{34}$ N. V. Smith, G. K. Wertheim, S. Hüfner, and M. M. Traum, Phys. Rev. B 10, 3197 (1974).

${ }^{35}$ N. V. Smith and L. F. Mattheiss, Phys. Rev. B 9, 1341 (1974); M. M. Traum and N. V. Smith, ibid. 9, 1353 (1974); N. V. Smith, ibid. 9, 1365 (1974).

${ }^{36} \mathrm{~K}$. Uosaki and H. Kita, in Modern Aspects of Electrochemistry, edited by R. E. White, J. O'M. Bockris, and B. E. Conway (Plenum, New York, 1986), Vol. 18, Chap. 1.

${ }^{37}$ A. Henglein, Ber. Bunsenges. Phys. Chem. 78, 1078 (1974).

${ }^{38}$ The nature of the solvation of the electron and the empty trap in the electrolyte solution must be evaluated to obtain precise information on the distribution function, and is not known at present. The actual distribution function may be entirely different from the Gaussian in the present system containing neutral species (Ref. 36).

${ }^{39}$ N. Kroo, Z. Szentirmay, and J. Felszerfalri, Phys. Status Solidi B 102, 227 (1980).

${ }^{40}$ H. Kanter, Phys. Rev. B 1, 522 (1970).

${ }^{41}$ H. Eyring, L. J. Walter, and G. E. Kimball, Quantum Chemistry (Wiley, New York, 1944), p. 114.

${ }^{42}$ K. Ohno, Quantum Physical Chemistry (in Japanese) (Tokyo University, Tokyo, 1989), Chap. 3.

${ }^{43}$ W. F. Krolikowski and W. E. Spicer, Phys. Rev. 185, 882 (1969).

${ }^{44}$ G. Denninger, V. Dose, and H. P. Bonzel, Phys. Rev. Lett. 48, 279 (1982).

45J. R. Manson and R. H. Ritchie, Phys. Rev. B 24, 4867 (1981).

${ }^{46}$ W. Drude and F. J. Himpsel, Phys. Rev. Lett. 60, 140 (1988).

${ }^{47}$ S. Ushioda, J. Lumin. 47, 131 (1991). 